\title{
Photoinduced Electron Pairing in a Driven Cavity
}

\author{
Hongmin Gao®, ${ }^{1, *}$ Frank Schlawin $\odot,{ }^{1, \dagger}$ Michele Buzzi®, ${ }^{2, \$}$ Andrea Cavalleri $\odot,{ }^{1,2,}$ and Dieter Jaksch $\odot^{1, \|}$ \\ ${ }^{1}$ Clarendon Laboratory, University of Oxford, Parks Road, Oxford OXI 3PU, United Kingdom \\ ${ }^{2}$ Max Planck Institute for the Structure and Dynamics of Matter, 22761 Hamburg, Germany
}

(Received 10 March 2020; accepted 5 June 2020; published 28 July 2020)

\begin{abstract}
We demonstrate how virtual scattering of laser photons inside a cavity via two-photon processes can induce controllable long-range electron interactions in two-dimensional materials. We show that laser light that is red (blue) detuned from the cavity yields attractive (repulsive) interactions whose strength is proportional to the laser intensity. Furthermore, we find that the interactions are not screened effectively except at very low frequencies. For realistic cavity parameters, laser-induced heating of the electrons by inelastic photon scattering is suppressed and coherent electron interactions dominate. When the interactions are attractive, they cause an instability in the Cooper channel at a temperature proportional to the square root of the driving intensity. Our results provide a novel route for engineering electron interactions in a wide range of two-dimensional materials including $A B$-stacked bilayer graphene and the conducting interface between $\mathrm{LaAlO}_{3}$ and $\mathrm{SrTiO}_{3}$.
\end{abstract}

DOI: 10.1103/PhysRevLett.125.053602

Introduction.-Engineering material properties on demand is widely considered as one of the central goals of modern condensed matter physics [1]. This can be achieved by static means, for instance by exfoliating materials into single atomic layers and combining them into van der Waals crystals [2], or by subjecting them to external stimuli such as pressure [3], strain [4,5], or external static fields. More recently, the dynamical manipulation of material properties by optical fields has gained much attention [6,7]. Strong laser pulses have been employed to induce metal-insulator transitions $[8,9]$ and synthetic magnetic fields [10], to melt striped phases [11-13], or charge density waves $[14,15]$. Strong excitation of specific phonon modes can even induce transient superconductinglike phases [13,16-19].

The coupling to shaped quantum vacua of cavities has been used to change molecular properties [20-24], reduce quasiparticle lifetimes in 2D electron gases [25] or drastically enhance the critical temperature of superconductors [26]. A growing number of theoretical works investigates this situation [27-33], where the competition between strong cavity and electron interactions has the potential to manifest in fascinating new physics. For example, in [32] some of us showed how the substantial subwavelength confinement of the cavity field in nanoplasmonic terahertz cavities [34-36] can give rise to cavity-mediated electron interactions. A significant drawback of this proposal is the lack of external dynamical experimental controllability of the properties of the interaction potential.

In this Letter, we combine adjustable external laser driving with the terahertz cavity's shaped vacuum fluctuations to overcome this limitation and create a novel type of controllable long-range electron interactions in 2D materials. Importantly, these interactions are manipulated by the parameters of the driving laser: their strength is proportional to the laser intensity $I_{d}$ and inversely proportional to the detuning $\delta_{c}=\omega_{c}-\omega_{L}\left(\omega_{c}\right.$ is the cavity frequency and $\omega_{L}$ is the driving frequency), which allows us to tune them to be attractive or repulsive by changing $\delta_{c}$. Intuitively they can be thought of as the result of inelastic scattering processes in which the intermediate state is dressed by the cavity. Strong coherent driving is essential to ensure that the two-photon diamagnetic scattering from which the interactions arise dominates over other (onephoton paramagnetic) processes, while the strong coupling to the cavity field is needed to enhance these interactions but not laser-induced heating processes. Another advantage of interactions arising from two-photon diamagnetic processes is that they can be induced in a wide range of materials. Here we consider a $2 \mathrm{D}$ electron gas as a prototypical system. We use parameters consistent with semiconductor quantum wells, gated bilayer graphene (BLG) [37] and the interface between lanthanum aluminate and strontium titanate $(\mathrm{LAO} /$ STO) [38] in the normal phase. The latter two are tunable 2D electron gases that can be adjusted arbitrarily close to a spontaneous superconducting instability and exhibit correlated electron phenomena, potentially making the physics even richer. Using these examples, we show that the interactions are long ranged and not effectively screened by the electrons. In the attractive regime, we find that they induce an instability in the Cooper channel at experimentally observable temperatures.

Setup.-We consider the setup shown in Fig. 1(a). A 2D electron system lies in the $x y$ plane inside a substrate material with relative permittivity $\epsilon_{r}$. It is coupled to the evanescent field of a complementary split-ring cavity which 


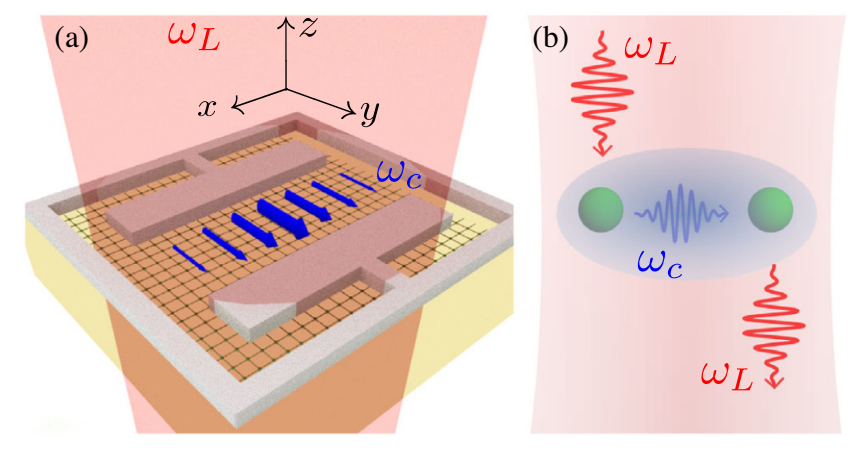

FIG. 1. (a) Setup: a 2D material (indicated by the square lattice) on a substrate (yellow) is coupled to a complementary split-ring cavity (gray) [39], whose electric field is represented by the blue arrows with thickness proportional to the field strength. The coupled system is driven by a laser field (schematically shown by the red shading) with wave vector $\mathbf{q}_{L} \| z$ axis and frequency $\omega_{L}$ detuned from the cavity frequency $\omega_{c}$. (b) The mechanism of inducing interactions between two electrons (green spheres). Laser (cavity) photons are represented by red (blue) wavy arrows and the green balls are electrons. The red (blue) shading symbolizes the laser (cavity) field.

sits on top of the substrate material, as, e.g., in Ref. [39]. This type of cavity is well described by a single-mode light field [40] and has been reported to exhibit strong vacuum fluctuations [41] due to its small cavity mode volume, $\mathcal{V}_{c}=\Lambda \lambda^{3}$, where $\lambda$ is the wavelength of the light mode in the substrate material and $\Lambda$ is the mode volume compression factor. Values of $\Lambda=2.5 / 8 \times 10^{-5}$ or even smaller have been reached in experiments and simulations [39,42]. It has also been demonstrated that the cavity is highly reflective only for a narrow bandwidth of frequencies near its resonance, $\omega_{c}$, and fairly transparent otherwise. We note that due to the small sizes of available bilayer graphene samples, LAO/STO may be more straightforwardly integrated into a cavity that extends several hundred microns in size. Furthermore, because LAO/STO structures are insulating at frequencies above the plasma frequency, dissipation may be managed better in these systems than in the graphene structures.

For simplicity, we thus model the cavity as supporting a single mode and perfectly transparent to all frequencies away from $\omega_{c}$. The cavity field is described by a vector potential $\mathbf{A}_{c}(\mathbf{r})=2 \mathbf{e}_{y} \sqrt{\hbar / \mathcal{V}_{c} \epsilon_{0} \epsilon_{r} \omega_{c}} \cos \left(q_{0} x\right)\left(b+b^{\dagger}\right)$, where $\mathbf{e}_{y}$ is the unit vector in the $y$ direction, $q_{0}=\omega_{c} \sqrt{\epsilon_{r}} / c$ with $c$ the speed of light in vacuum, $\epsilon_{0}$ the vacuum permittivity and $b$ the bosonic annihilation operator for the cavity photon. The cavity-matter system is driven by a strong laser that we describe by an oscillating classical field with vector potential $\mathbf{A}_{d}(t) \propto \mathbf{e}_{y} \sqrt{I_{d}} \sin \left(\left|\mathbf{q}_{L}\right| z-\omega_{L} t\right)$, where $\mathbf{q}_{L}$ is the laser photon wave vector and $t$ is the time. The laser frequency $\omega_{L}=\omega_{c}-\delta_{c}$ lies outside of the cavity reflectivity window, such that the driving field interacts directly with the electronic system.
The coupled cavity-electron Hamiltonian in the Coulomb gauge reads [43],

$H=\sum_{j} \frac{\left[\boldsymbol{p}_{j}+e \mathbf{A}_{\mathrm{tot}}\left(\boldsymbol{r}_{j}, t\right)\right]^{2}}{2 m}+V_{b g}\left(\boldsymbol{r}_{j}\right)+H_{\mathrm{Coul}}+H_{\mathrm{cav}}$,

with $\mathbf{A}_{\text {tot }}\left(\boldsymbol{r}_{j}, t\right)=\mathbf{A}_{c}\left(\boldsymbol{r}_{j}\right)+\mathbf{A}_{d}\left(\boldsymbol{r}_{j}, t\right)$. Here $\boldsymbol{p}_{j}$ and $\boldsymbol{r}_{j}$ are the momentum and position operators of electron $j$ with bare mass $m, V_{b g}$ is the static potential due to the positive background, $H_{\text {Coul }}$ is the Coulomb interaction between the electrons and $H_{\text {cav }}=\hbar \omega_{c} b^{\dagger} b$ is the unperturbed cavity Hamiltonian. We assume that the single-electron physics arising from the first part of the Hamiltonian [Eq. (1)] is described by a single band and higher bands are far detuned from the cavity frequencies considered here $(\lesssim 10 \mathrm{THz})$ such that dynamical Stark effects are negligible. The first term in Eq. (1) also yields one-photon paramagnetic $(\boldsymbol{p} \cdot \mathbf{A})$ and two-photon diamagnetic $\left(\mathbf{A}^{2}\right)$ interactions between the transverse light fields and the electrons. In Eq. (1) we neglected the explicit description of environmental EM field modes, though we checked that for the parameters we consider their main influence-inelastic scattering of laser photons into the environment-does not heat the electrons significantly. This is because the engineered interactions scale as $1 /\left(\Lambda \delta_{c}\right)$ [see Eq. (3)] whereas the inelastic scattering rate scales as $1 / \omega_{L} \ll 1 /\left(\Lambda \delta_{c}\right)$ [43] and thus affects the electron dynamics only very weakly.

Rotating-frame transformation.-Within the single electronic band, the one-photon processes which normally dominate the optical response of materials are far offresonant. However, the two-photon diamagnetic interactions include near-resonant processes [schematically shown in Fig. 1(b)] in which laser photons are scattered into the cavity. When the driving is strong [43], these processes become the principal coupling mechanism of the electrons to the cavity field fluctuations and dominate the dynamics. This becomes clear when we move into a frame corotating with the laser frequency $\omega_{L}$ using the unitary transformation $U(t)=\exp \left(-i \omega_{L} t b^{\dagger} b\right)$, see Fig. 2(a). This transformation does not affect the electronic Hamiltonian but changes $\omega_{c}$ in $H_{\text {cav }}$ to $\delta_{c}\left(\left|\delta_{c}\right| \ll \omega_{c}\right)$ and removes the explicit time dependence of the near-resonant two-photon diamagnetic interactions. In this frame, all the one-photon interaction terms oscillate rapidly at $\omega_{L}$, thus their effect on the electrons is proportional to $\sim 1 / \omega_{L}$.

In contrast, the effect of two-photon diamagnetic interactions (apart from an irrelevant energy shift) is proportional to $\sim 1 / \delta_{c} \gg 1 / \omega_{L}$. In this rotating frame, the nearresonant part of the two-photon diamagnetic interactions is given by

$$
H_{\text {light-matter }}=\sum_{\mathbf{q}= \pm q_{0} \mathbf{e}_{x}} \frac{g_{0}}{\sqrt{\mathcal{S}}} \varrho_{\mathbf{q}}\left(b+b^{\dagger}\right)
$$




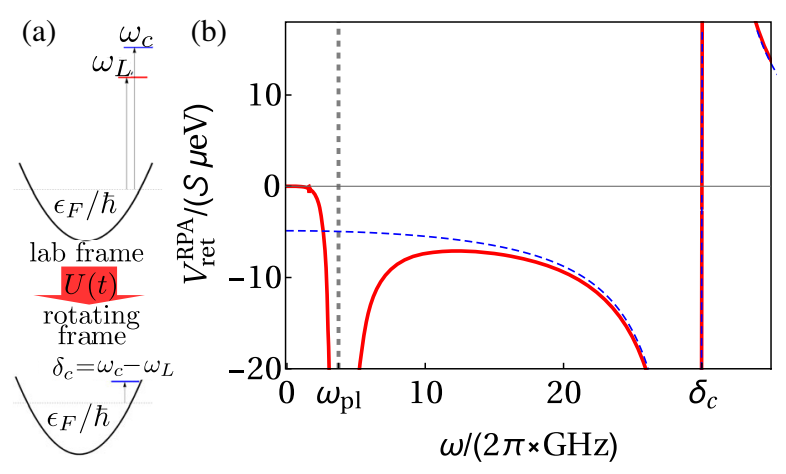

FIG. 2. (a) Relevant energy levels for the two-photon diamagnetic interactions involving the driving (red) and cavity (blue) fields in the lab frame (top) and in a rotating frame (bottom). The electron dispersion is plotted in black. $\epsilon_{F}$ is the Fermi level. (b) The real part of the screened interaction potential $V_{\text {ret }}^{\mathrm{RPA}} / \mathcal{S}$ is shown in red. The real part of $V_{\text {ret }}^{\text {bare }} / \mathcal{S}$ (blue dashed line) is shown for comparison. The plasmon resonance $\omega_{\mathrm{pl}}\left(q_{0}\right)$ is indicated by the grey vertical dashed line. The small irregularity below $\omega_{\mathrm{pl}}$ comes from the discontinuity of the $2 \mathrm{D}$ electron polarizability given in Ref. [59]. The cavity parameters used are $\omega_{c}=2 \pi \times 0.3 \mathrm{THz}, \delta_{c}=0.1 \omega_{c}$, quality factor $Q=500$ and $\Lambda=2.5 / 8 \times 10^{-5}$. We set $\mathcal{S} \approx 2.1 \times 10^{-5} \lambda^{2}$, where the proportionality constant is obtained from Ref. [39]. The driving intensity is $I_{d}=3 \mathrm{MW} \mathrm{cm}^{-2}$. We use an electron density $n_{e}=10^{12} \mathrm{~cm}^{-2}$ and $m^{*}=2 m$ consistent with various $2 \mathrm{D}$ materials such at (gated) LAO/STO and monolayer TMDs [60-62]. The plasmon damping rate is set to $10 \%$ of plasmon frequency [63]. We consider a $\mathrm{SrTiO}_{3}$ substrate with a large $\epsilon_{r} \sim 10^{4}$ at low temperatures [64].

where $\mathcal{S}$ is the cavity area in the $x y$ plane and $\varrho_{\mathbf{q}}=\sum_{\mathbf{k}, \sigma} c_{\mathbf{k}+\mathbf{q}, \sigma}^{\dagger} c_{\mathbf{k}, \sigma}$ is the electron area density in $k$ space. $c_{\mathbf{k}, \sigma}$ annihilates an electron with quasimomentum $\mathbf{k}$ and spin $\sigma$. The interaction vertex reads $g_{0}=$ $\alpha \sqrt{I_{d} \hbar^{3} \omega_{c}^{2} \mathcal{S} /\left(2 \pi m^{2} \Lambda \omega_{L}^{2} c^{2}\right)}$, where $\alpha$ is the fine-structure constant.

Cavity-mediated interactions. - The light-matter coupling given by Eq. (2) yields effective electron interactions through the process depicted in Fig. 1(b): a laser photon scatters virtually off an electron into the cavity, where it gets rescattered by a second electron back into the laser beam. The fact that the two-photon diamagnetic interactions couple to the electron density [see Eq. (2)] allows us to apply some of the intuition from other boson-exchange interactions [65]. Specifically, it means the exchange of virtual cavity photons (with effective frequencies $\delta_{c}$ in the rotating frame) mediates density-density interactions between the electrons. The interactions will be attractive (repulsive) when $\delta_{c}>0(<0)$, i.e., the driving laser is red (blue) detuned from the cavity.

The bare retarded interaction created by the exchange of cavity photons reads $V_{\text {ret }}^{\text {bare }}(\mathbf{q}, \omega+i \eta)=\left|g_{0}\right|^{2} D^{(0)}(\mathbf{q}, \omega+i \eta)$, where $\eta=0^{+}$and $D^{(0)}$ is the bare cavity photon Green's function in the rotating frame. In the static limit $\omega=0$, we recover the effective interaction Hamiltonian obtained using a Schrieffer-Wolff transformation, $H_{\text {int }}=(1 / 2 \mathcal{S}) \times$ $\sum_{\mathbf{q}} V^{(0)}(\mathbf{q}) \varrho_{\mathbf{q}} Q_{-\mathbf{q}}$, where the expression for the bare interaction potential,

$$
\frac{V^{(0)}(\mathbf{q})}{\mathcal{S}}=\frac{\alpha^{2} \hbar^{2}}{\pi c^{2} m^{2}} \frac{\omega_{c}^{2}}{\omega_{L}^{2}} \frac{I_{\mathrm{d}}}{\Lambda \delta_{c}} \delta_{ \pm \mathbf{q}, q_{0} \mathbf{e}_{x}},
$$

confirms our intuition that the interaction strength is proportional to the driving intensity and can be tuned to be attractive or repulsive by the detuning. We note that this potential only depends on the momentum transferred between the two electrons and does not depend on the electron dispersion. Moreover, $V^{(0)}(\mathbf{q})$ is very localized in momentum space because the photon momenta are much smaller than electronic ones. Hence, this electron interaction is long ranged in real space. In the following section, we will investigate how it is screened by the twodimensional electron gas.

Screening.-Within the random phase approximation (RPA), the screened interaction potential in imaginary time formalism reads [48],

$$
V^{\mathrm{RPA}}(\tilde{q})=\frac{\left|g^{\mathrm{RPA}}(\tilde{q})\right|^{2} D^{(0)}(\tilde{q})}{1-\left|g_{0}\right|^{2} \chi^{\mathrm{RPA}}(\tilde{q}) D^{(0)}(\tilde{q})},
$$

where we have used the four-vector notation with $\tilde{q} \equiv$ $\left\{\mathbf{q}, i \nu_{n}\right\}$ at momentum $\mathbf{q}$ and the bosonic Matsubara frequency, $i \nu_{n}=2 \pi n k_{B} T / \hbar, n \in \mathbb{Z}$. $k_{B}$ is the Boltzmann constant and $T$ is the temperature. $\chi^{\mathrm{RPA}}$ is the screened polarizability of the two-dimensional electron gas, and $g^{\mathrm{RPA}}$ is the screened vertex of Eq. (2). We obtain $\chi^{\mathrm{RPA}}$ and $g^{\mathrm{RPA}}$ for a parabolic band with an effective mass $m^{*}$ using results calculated in [59] and we present their expressions in the Supplemental Material [43]. Since $q_{0} \ll k_{f}$, we are in the long-wavelength limit where only electrons near the Fermi surface contribute to screening. Thus, the results in [59] are valid in this limit for other single band dispersions [66]. The retarded interaction is obtained from Eq. (4) (a function in imaginary frequency) by analytic continuation to real frequencies [53]: $V_{\text {ret }}^{\mathrm{RPA}}(\omega)=V^{\mathrm{RPA}}\left( \pm q_{0} \mathbf{e}_{x}, i \nu_{n} \rightarrow \omega+i \eta\right)$.

We show an example of the real part of the screened retarded interaction potential as a function of frequency in Fig. 2(b). Here we also included finite Drude-like electron $[49,50]$ and cavity damping rates (see [43] for details). Evidently, contributions to the interaction at small frequencies are strongly screened. The precise choice of the damping rates alters only the size of the dips at $\omega=\omega_{\mathrm{pl}}$ and $\delta_{c}$ but not the interaction potential in the broad region of frequency in between, thus it has little effect on the physics. In the present case where $\delta_{c}>0$ the attractive cavity-mediated interaction softens the plasmons and the cavity frequency is weakly blue shifted. This effect only becomes appreciable at very large laser intensities, and 


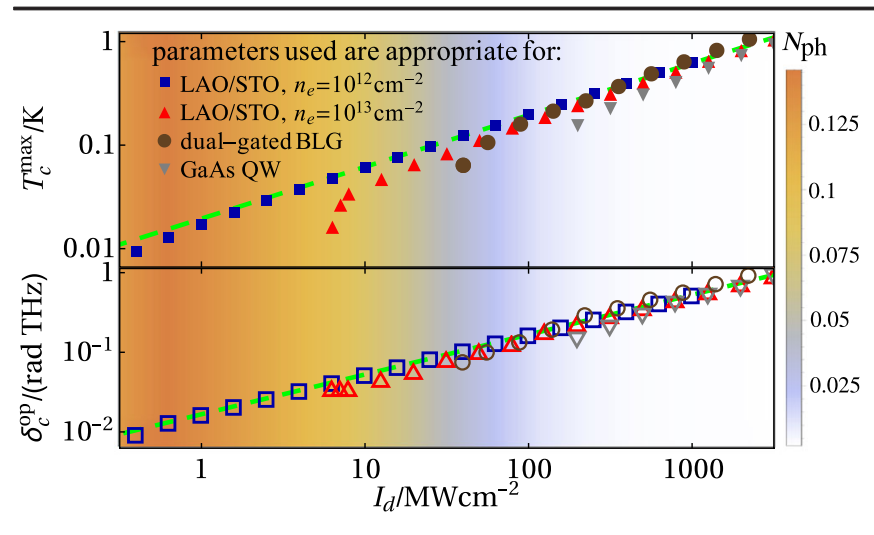

FIG. 3. Maximum $T_{c} \mathrm{~s}$ (with respect to driving and cavity parameters) for the Cooper channel instability (top panel) and the optimal detunings, $\delta_{c}^{\text {op }}$, (bottom panel) are plotted for a range of driving intensities. We calculate them for four sets of electron parameters. All of them follow the dashed green line (same in both panels) representing Eq. (5) at large enough driving intensities. The color map shows the cavity photon number $N_{\mathrm{ph}}$ for different $I_{d}$ with the optimal driving and cavity parameters at $T_{c}^{\max }$ using electron and substrate parameters appropriate for gated LAO/STO [same as those used in Fig. 2(b)]. We used $n_{e}=3 \times 10^{11}, 5 \times 10^{10} \mathrm{~cm}^{-2}, \epsilon_{r}=10,13$ for dual-gated BLG and GaAs based quantum well $(\mathrm{QW})$, respectively $[36,68,69]$; $m^{*}=0.07 m$ for GaAs QW [36]. $A B$-stacked BLG dispersion is calculated as in Ref. [70] with a gate-induced band gap of $50 \mathrm{meV}$. We used $\omega_{c}=10 \delta_{c}^{\mathrm{op}}$.

cannot be seen in Fig. 2(b). Crucially, however, for frequencies between the plasmon resonance and the detuning $\delta_{c}$, the interaction remains attractive (repulsive for $\left.\delta_{c}<0\right)$ and is hardly affected by screening. We can understand this behavior by investigating the expression for the screened interaction in more detail.

Since we are in the long-wavelength limit, we have $\chi^{\mathrm{RPA}} \propto q_{0}^{2}$. Physically this means the electrons are too slow to screen the fast oscillating electromagnetic fields and hence the cavity-mediated interactions can only be screened weakly. Corrections to the bare interaction become appreciable when $\omega$ approaches the plasmon resonance of the system. In a two-dimensional electron gas, the plasmon dispersion is given by $\omega_{\mathrm{pl}}^{2}\left(q_{0}\right)=e^{2} k_{f} v_{f} q_{0} /\left(4 \pi \hbar \epsilon_{r} \epsilon_{0}\right)$ [67], where $v_{f}$ is the Fermi velocity. This yields plasmon frequencies in the range $\omega_{\mathrm{pl}} / 2 \pi \sim 4 \mathrm{GHz}$ [for the parameters of Fig. 2(b)]. Hence, it is only relevant at temperatures below $T \simeq 30 \mathrm{mK}$, as we will see in Fig. 3. At higher temperatures, the Matsubara frequencies will not cover this low-frequency region and the cavity-mediated interactions cannot be screened effectively by the electron gas. They remain long-ranged interactions that can be imposed externally on the two-dimensional electronic system.

Cooper channel instability.-To gauge the strength of the cavity-mediated interactions that could be achieved experimentally, we consider the case of attractive interactions and study the Cooper instability they induce. This pairing instability occurs when a pair of electrons with opposite momenta and frequencies attract and repeatedly scatter off one another to cause a divergence of the pair scattering vertex $\Gamma(\tilde{k} ; \tilde{p})$, where $\tilde{k}(\tilde{p})$ is the incoming (outgoing) electron four-momentum [48,52]. The instability could potentially be detected with techniques such as transport or magnetization measurements [25,54,71]. $\Gamma(\tilde{k} ; \tilde{p})$ is obtained by solving a Dyson equation [43,48]. As the photon momentum is very small compared to electronic momenta, we approximate the exact interaction by a $\delta$-function potential in $k$ space, $V^{\mathrm{RPA}}(\tilde{q}) \propto \delta(\mathbf{q})$, which simplifies the internal summations in the pair scattering ladder diagrams, yielding a matrix equation (see [43] for more details) $\Gamma\left(i k_{n} ; i p_{n}\right)=-V^{\mathrm{RPA}}\left(i k_{n}-i p_{n}\right)+$ $\mathcal{M}\left(i k_{n} ; i \nu_{n}\right) \Gamma\left(i \nu_{n} ; i p_{n}\right), \quad$ where $\quad \mathcal{M}\left(i k_{n} ; i \nu_{n}\right)=$ $-2 V^{\mathrm{RPA}}\left(i k_{n}-i \nu_{n}\right) G^{(0)}\left(i \nu_{n}\right) G^{(0)}\left(-i \nu_{n}\right) /(\mathcal{S} \beta)$, and $G^{(0)}$ is the bare electron Green's function and $\beta=1 / k_{B} T$ is the inverse temperature. We then numerically compute the temperature $T_{c}$, at which the pair scattering vertex diverges using the Dyson equation with the screened interaction potential within RPA for different choices of driving and cavity parameters.

$T_{c}$ depends on the driving intensity, the cavity frequency and the detuning. In the following, we vary the detuning to obtain the highest critical temperature for fixed intensities. The results are shown in Fig. 3, where the highest $T_{c}, T_{c}^{\max }$ (top panel), and the corresponding detuning $\delta_{c}^{\mathrm{op}}$ (bottom panel) are plotted against the intensity for four sets of electron and substrate parameters. We find that provided $\omega_{c} \gg \delta_{c}$ and $\delta_{c}$ is well above the plasmon resonance $T_{c}$ depends very weakly on $\omega_{c}$ once we fix $\delta_{c}$ : a smaller $\omega_{c}$ raises $T_{c}$ very slightly. We remark that the necessary intensities to induce $T_{c} \sim 1 \mathrm{~K}$ are experimentally achievable with pulsed THz light sources [9,72-76]. At these high intensities, laser-induced heating of the substrate material becomes relevant [43], such that a pulse driving protocol might become necessary. The green dashed line represents a function $\propto \sqrt{I_{d}}$ and it fits the $T_{c}^{\max }$ and the $\delta_{c}^{\mathrm{op}}$ points very well at high driving intensities,

$$
T_{c}^{\mathrm{max}} / \mathrm{K} \approx \delta_{c}^{\mathrm{op}} \mathrm{ps} / \mathrm{rad} \approx 10^{-4.5} \sqrt{I_{d} /\left(\Lambda \mathrm{MW} \mathrm{cm}^{-2}\right)} .
$$

The numerical constants depend only on fundamental constants. The deviations of our numerical results in Fig. 3 from Eq. (5) at smaller intensities are caused by the screening due to plasmons which reduces $T_{c}^{\max }$. Consequently, the onset of this deviation is determined by the plasmon frequency and hence the substrate dielectric constant, the 2D electron density and the Fermi velocity. The materials with fewer data points in Fig. 3 have higher plasmon resonances, so the screening is stronger for them, causing $T_{c}^{\max }$ to drop quickly to 0 at higher intensities than materials with lower plasmon resonances. 
Figure 3 shows that in order to achieve the highest critical temperature at a given driving intensity, one must choose an appropriate detuning (and hence cavity and driving frequencies). We understand this by applying an approximation to the Dyson equation to estimate the Cooper instability temperature. The approximation, which underestimates $T_{c}$ by only a few percent, amounts to retaining only the two fermionic Matsubara frequencies of the lowest magnitude in the Dyson equation [48]. This allows us to extract the critical temperature analytically, $k_{B} T_{c} \approx-2 \pi^{-2} V^{\mathrm{RPA}}\left(q_{0} \mathbf{e}_{x}, 2 i \pi k_{B} T_{c} / \hbar\right) / \mathcal{S}$. Above the plasmon resonance, the interaction is effectively unscreened so for a perfect cavity $V^{\mathrm{RPA}} \propto I_{d} \delta_{c} /\left[\left(2 \pi k_{B} T_{c} / \hbar\right)^{2}+\delta_{c}^{2}\right]$ [43]. For all values of $T_{c}$, this is maximized when we choose $\delta_{c}^{\mathrm{op}}=2 \pi k_{B} T_{c} / \hbar$. We substitute the two relations above back into the approximate equation for the critical temperature to obtain $T_{c}^{\max } \propto \sqrt{I_{d}}$ consistent with Fig. 3 and Eq. (5). We note that a finite cavity decay rate reduces $\delta_{c}^{\mathrm{op}}$ only slightly.

We furthermore verified that the cavity photon population $N_{\text {ph }}$ remains low at $T_{c}$ (see color bar in Fig. 3) when we use the optimal cavity and driving parameters [43]. This justifies neglecting two-photon diamagnetic interactions involving only the cavity modes $\left(\mathbf{A}_{c}^{2}\right.$ terms). The decrease of $N_{\text {ph }}$ with higher driving intensity is due to the blue-shift of the cavity resonance (see the above discussion on screening) resulting in a lower thermal cavity population. Finally, we note that keeping $\delta_{c}$ well above the cavity linewidth and the plasmon frequency suppresses the effect of cavity-induced heating or cooling [77].

Conclusions.-We showed that the combination of external driving and strong coupling to a cavity induces controllable long-range electron interactions in single-band 2D materials. Provided that the driving is detuned from the cavity further than the plasmon frequencies at the relevant wave vectors, the interactions cannot be effectively screened. The interactions are generated by two-photon diamagnetic processes. Therefore, they are largely independent of the electron band dispersion and can be induced on top of existing interactions. These induced interactions could be used to control or enhance existing instabilities or give rise to different quantum states through competition with other short-range interactions [78-81]. To date, engineered long-range quantum fluctuation-mediated interactions have mainly been studied in the context of ultracold atoms [78,82-93]. Our proposal potentially opens the possibility to explore a vast range of novel physics related to unscreened controllable long-range interactions in condensed matter setups.

We would like to thank A. Imamoğlu, J. Faist, G. Mazza, and C. Sánchez Muñoz for useful discussions. This work has been supported by the European Research Council under the European Union's Seventh Framework Programme (FP7/2007-2013)/ERC Grant Agreement
No. 319286 Q-MAC and by EPSRC Grant No. EP/ P009565/1. We acknowledge support from the Deutsche Forschungsgemeinschaft (DFG) via the Cluster of Excellence "CUI: Advanced Imaging of Matter" (EXC 2056 - Project ID 390715994) and the Collaborative Research Center SFB 925.

*hongmin.gao@physics.ox.ac.uk

frank.schlawin@physics.ox.ac.uk

†michele.buzzi@mpsd.mpg.de

§andrea.cavalleri@mpsd.mpg.de

"dieter.jaksch@physics.ox.ac.uk

[1] D. N. Basov, R. D. Averitt, and D. Hsieh, Towards properties on demand in quantum materials, Nat. Mater. 16, 1077 (2017).

[2] K. S. Novoselov, A. Mishchenko, A. Carvalho, and A. H. Castro Neto, 2D materials and van der Waals heterostructures, Science 353, aac9439 (2016).

[3] J. V. Badding, J. F. Meng, and D. A. Polvani, Pressure tuning in the search for new and improved solid state materials, Chem. Mater. 10, 2889 (1998).

[4] D. G. Schlom, L.-Q. Chen, C.-B. Eom, K. M. Rabe, S. K. Streiffer, and J.-M. Triscone, Strain tuning of ferroelectric thin films, Annu. Rev. Mater. Res. 37, 589 (2007).

[5] P. Zubko, S. Gariglio, M. Gabay, P. Ghosez, and J.-M. Triscone, Interface physics in complex oxide heterostructures, Annu. Rev. Condens. Matter Phys. 2, 141 (2011).

[6] R. Mankowsky, M. Först, and A. Cavalleri, Non-equilibrium control of complex solids by nonlinear phononics, Rep. Prog. Phys. 79, 064503 (2016).

[7] D. Nicoletti and A. Cavalleri, Nonlinear light-matter interaction at terahertz frequencies, Adv. Opt. Photonics 8, 401 (2016).

[8] M. Rini, R. Tobey, N. Dean, J. Itatani, Y. Tomioka, Y. Tokura, R. W. Schoenlein, and A. Cavalleri, Control of the electronic phase of a manganite by mode-selective vibrational excitation, Nature (London) 449, 72 (2007).

[9] M. Liu, H. Y. Hwang, H. Tao, A. C. Strikwerda, K. Fan, G. R. Keiser, A. J. Sternbach, K. G. West, S. Kittiwatanakul, J. Lu, S. A. Wolf, F. G. Omenetto, X. Zhang, K. A. Nelson, and R. D. Averitt, Terahertz-field-induced insulator-tometal transition in vanadium dioxide metamaterial, Nature (London) 487, 345 (2012).

[10] T. F. Nova, A. Cartella, A. Cantaluppi, M. Först, D. Bossini, R. V. Mikhaylovskiy, A. V. Kimel, R. Merlin, and A. Cavalleri, An effective magnetic field from optically drivenÂ phonons, Nat. Phys. 13, 132 (2016).

[11] D. Fausti, R. I. Tobey, N. Dean, S. Kaiser, A. Dienst, M. C. Hoffmann, S. Pyon, T. Takayama, H. Takagi, and A. Cavalleri, Light-induced superconductivity in a stripe-ordered cuprate, Science 331, 189 (2011).

[12] M. Först, R. I. Tobey, H. Bromberger, S. B. Wilkins, V. Khanna, A. D. Caviglia, Y.-D. Chuang, W. S. Lee, W. F. Schlotter, J. J. Turner, M. P. Minitti, O. Krupin, Z. J. Xu, J. S. Wen, G. D. Gu, S. S. Dhesi, A. Cavalleri, and J. P. Hill, Melting of Charge Stripes in Vibrationally Driven $\mathrm{La}_{1.875} \mathrm{Ba}_{0.125} \mathrm{CuO}_{4}$ : Assessing the Respective Roles of 
Electronic and Lattice Order in Frustrated Superconductor, Phys. Rev. Lett. 112, 157002 (2014).

[13] K. A. Cremin, J. Zhang, C. C. Homes, G. D. Gu, Z. Sun, M. M. Fogler, A. J. Millis, D. N. Basov, and R. D. Averitt, Photoenhanced metastable c-axis electrodynamics in stripeordered cuprate $\mathrm{La}_{1.885} \mathrm{Ba}_{0.115} \mathrm{CuO}_{4}$, Proc. Natl. Acad. Sci. U.S.A. 116, 19875 (2019).

[14] M. Först, A. Frano, S. Kaiser, R. Mankowsky, C. R. Hunt, J. J. Turner, G. L. Dakovski, M. P. Minitti, J. Robinson, T. Loew, M. Le Tacon, B. Keimer, J. P. Hill, A. Cavalleri, and S.S. Dhesi, Femtosecond $x$ rays link melting of chargedensity wave correlations and light-enhanced coherent transport in $\mathrm{YBa}_{2} \mathrm{Cu}_{3} \mathrm{O}_{6.6}$, Phys. Rev. B 90, 184514 (2014).

[15] R. Mankowsky, B. Liu, S. Rajasekaran, H. Y. Liu, D. Mou, X. J. Zhou, R. Merlin, M. Först, and A. Cavalleri, Dynamical Stability Limit for the Charge Density Wave in $\mathrm{K}_{0.3} \mathrm{MoO}_{3}$, Phys. Rev. Lett. 118, 116402 (2017).

[16] W. Hu, S. Kaiser, D. Nicoletti, C. R. Hunt, I. Gierz, M. C. Hoffmann, M. Le Tacon, T. Loew, B. Keimer, and A. Cavalleri, Optically enhanced coherent transport in $\mathrm{YBa}_{2} \mathrm{Cu}_{3} \mathrm{O}_{6.5}$ by ultrafast redistribution of interlayer coupling, Nat. Mater. 13, 705 (2014).

[17] R. Mankowsky, A. Subedi, M. Först, S. O. Mariager, M. Chollet, H. T. Lemke, J. S. Robinson, J. M. Glownia, M. P. Minitti, A. Frano, M. Fechner, N. A. Spaldin, T. Loew, B. Keimer, A. Georges, and A. Cavalleri, Nonlinear lattice dynamics as a basis for enhanced superconductivity in $\mathrm{YBa}_{2} \mathrm{Cu}_{3} \mathrm{O}_{6.5}$, Nature (London) 516, 71 (2014).

[18] M. Mitrano, A. Cantaluppi, D. Nicoletti, S. Kaiser, A. Perucchi, S. Lupi, P. Di Pietro, D. Pontiroli, M. Ricco, S. R. Clark, D. Jaksch, and A. Cavalleri, Possible lightinduced superconductivity in $\mathrm{K}_{3} \mathrm{C}_{60}$ at high temperature, Nature (London) 530, 461 (2016).

[19] M. Budden, T. Gebert, M. Buzzi, G. Jotzu, E. Wang, T. Matsuyama, G. Meier, Y. Laplace, D. Pontiroli, M. Riccò, F. Schlawin, D. Jaksch, and A. Cavalleri, Evidence for metastable photo-induced superconductivity in $\mathrm{K}_{3} \mathrm{C}_{60}$, arXiv:2002.12835.

[20] A. Thomas, J. George, A. Shalabney, M. Dryzhakov, S. J. Varma, J. Moran, T. Chervy, X. Zhong, E. Devaux, C. Genet, J. A. Hutchison, and T. W. Ebbesen, Ground-state chemical reactivity under vibrational coupling to the vacuum electromagnetic field, Angew. Chem. Int. Ed. 55, 11462 (2016).

[21] A. Thomas, L. Lethuillier-Karl, K. Nagarajan, R. M. A. Vergauwe, J. George, T. Chervy, A. Shalabney, E. Devaux, C. Genet, J. Moran, and T. W. Ebbesen, Tilting a groundstate reactivity landscape by vibrational strong coupling, Science 363, 615 (2019).

[22] T. W. Ebbesen, Hybrid light-matter states in a molecular and material science perspective, Acc. Chem. Res. 49, 2403 (2016).

[23] J. Feist, J. Galego, and F. J. Garcia-Vidal, Polaritonic chemistry with organic molecules, ACS Photonics 5, 205 (2018).

[24] M. Ruggenthaler, J. Flick, H. Appel, and A. Rubio, From a quantum-electrodynamical light-matter description to novel spectroscopies, Nat. Rev. Chem. 2, 0118 (2018).

[25] G. L. Paravicini-Bagliani, F. Appugliese, E. Richter, F. Valmorra, J. Keller, M. Beck, N. Bartolo, C. Rössler,
T. Ihn, K. Ensslin, C. Ciuti, G. Scalari, and J. Faist, Magneto-transport controlled by Landau polariton states, Nat. Phys. 15, 186 (2019).

[26] A. Thomas, E. Devaux, K. Nagarajan, T. Chervy, M. Seidel, D. Hagenmüller, S. Schütz, J. Schachenmayer, C. Genet, G. Pupillo, and T. W. Ebbesen, Exploring superconductivity under strong coupling with the vacuum electromagnetic field, arXiv:1911.01459.

[27] J. B. Curtis, Z. M. Raines, A. A. Allocca, M. Hafezi, and V. M. Galitski, Cavity quantum eliashberg enhancement of superconductivity, Phys. Rev. Lett. 122, 167002 (2019).

[28] M. A. Sentef, M. Ruggenthaler, and A. Rubio, Cavity quantum-electrodynamical polaritonically enhanced electron-phonon coupling and its influence on superconductivity, Sci. Adv. 4, eaau6969 (2018).

[29] M. Kiffner, J. R. Coulthard, F. Schlawin, A. Ardavan, and D. Jaksch, Manipulating quantum materials with quantum light, Phys. Rev. B 99, 085116 (2019).

[30] G. Mazza and A. Georges, Superradiant Quantum Materials, Phys. Rev. Lett. 122, 017401 (2019).

[31] A. A. Allocca, Z. M. Raines, J. B. Curtis, and V. M. Galitski, Cavity superconductor-polaritons, Phys. Rev. B 99, 020504 (R) (2019).

[32] F. Schlawin, A. Cavalleri, and D. Jaksch, Cavity-mediated electron-photon superconductivity, Phys. Rev. Lett. 122, 133602 (2019).

[33] M. Kiffner, J. Coulthard, F. Schlawin, A. Ardavan, and D. Jaksch, Mott polaritons in cavity-coupled quantum materials, New J. Phys. 21, 073066 (2019).

[34] G. Scalari, C. Maissen, D. Turčinková, D. Hagenmüller, S. De Liberato, C. Ciuti, C. Reichl, D. Schuh, W. Wegscheider, M. Beck, and J. Faist, Ultrastrong coupling of the cyclotron transition of a $2 \mathrm{D}$ electron gas to a $\mathrm{THz}$ metamaterial, Science 335, 1323 (2012).

[35] S. Smolka, W. Wuester, F. Haupt, S. Faelt, W. Wegscheider, and A. Imamoglu, Cavity quantum electrodynamics with many-body states of a two-dimensional electron gas, Science 346, 332 (2014).

[36] Q. Zhang, M. Lou, X. Li, J. L. Reno, W. Pan, J. D. Watson, M. J. Manfra, and J. Kono, Collective non-perturbative coupling of 2D electrons with high-quality-factor terahertz cavity photons, Nat. Phys. 12, 1005 (2016).

[37] Y. Cao, V. Fatemi, S. Fang, K. Watanabe, T. Taniguchi, E. Kaxiras, and P. Jarillo-Herrero, Unconventional superconductivity in magic-angle graphene superlattices, Nature (London) 556, 43 (2018).

[38] A. D. Caviglia, S. Gariglio, N. Reyren, D. Jaccard, T. Schneider, M. Gabay, S. Thiel, G. Hammerl, J. Mannhart, and J.-M. Triscone, Electric field control of the $\mathrm{LaAlO}_{3} / \mathrm{SrTiO}_{3}$ interface ground state, Nature (London) 456, 624 (2008).

[39] C. Maissen, G. Scalari, F. Valmorra, M. Beck, J. Faist, S. Cibella, R. Leoni, C. Reichl, C. Charpentier, and W. Wegscheider, Ultrastrong coupling in the near field of complementary split-ring resonators, Phys. Rev. B 90, 205309 (2014).

[40] The next higher mode has a frequency of roughly $4 \omega_{c}$ and is more lossy [39].

[41] J. Keller, G. Scalari, S. Cibella, C. Maissen, F. Appugliese, E. Giovine, R. Leoni, M. Beck, and J. Faist, Few-Electron 
Ultrastrong Light-Matter Coupling at $300 \mathrm{GHz}$ with Nanogap Hybrid LC Microcavities, Nano Lett. 17, 7410 (2017).

[42] N. Kim, S. In, D. Lee, J. Rhie, J. Jeong, D.-S. Kim, and N. Park, Colossal terahertz field enhancement using splitring resonators with a sub-10 nm gap, ACS Photonics 5, 278 (2018).

[43] See the Supplemental Material at http://link.aps.org/ supplemental/10.1103/PhysRevLett.125.053602 for derivation of full system Hamiltonian, which includes Refs. [44,45], discussions of the different interaction terms along with the required driving intensity for the diamagnetic interactions to dominate the dynamics which includes Refs. [32,39,46,47], RPA screening calculations, which includes Refs. [48-51], the Dyson equation for the pair scattering vertex, which includes Ref. [52], how to calculate the cavity photon population, which includes Ref. [53], and discussion on laser-induced heating of the substrate material, which includes Refs. [54-58].

[44] M. Kira and S. W. Koch, Semiconductor Quantum Optics (Cambridge University Press, Cambridge, England, 2011).

[45] J. Li, D. Golez, G. Mazza, A. J. Millis, A. Georges, and M. Eckstein, Electromagnetic coupling in tight-binding models for strongly correlated light and matter, Phys. Rev. B 101, 205140 (2020).

[46] M. Kira and S. W. Koch, Interactions in Semiconductors, in Semiconductor Quantum Optics (Cambridge University Press, Cambridge, England, 2011), pp. 253-278.

[47] E. J. Sie, J. W. McIver, Y.-H. Lee, L. Fu, J. Kong, and N. Gedik, Optical Stark effect in 2D semiconductors, Proc. SPIE Int. Soc. Opt. Eng. 9835, 129 (2016).

[48] H. Bruus and K. Flensberg, Many-Body Quantum Theory in Condensed Matter Physics-An Introduction (Oxford University Press, United States, 2004).

[49] M. Fox, , Optical Properties of Solids, Oxford Master Series in Condensed Matter Physics (Oxford University Press, Oxford, 2001).

[50] C. Kittel, , Quantum Theory of Solids (John Wiley \& Sons, New York, 1987).

[51] F. Stern and W. E. Howard, Properties of semiconductor surface inversion layers in the electric quantum limit, Phys. Rev. 163, 816 (1967).

[52] A. A. Abrikosov, Methods of Quantum Field Theory in Statistical Physics (Dover Publications, New York, 1975).

[53] G. Mahan, , Many-Particle Physics, Physics of Solids and Liquids (Springer US, New York, 2000).

[54] J. W. McIver, B. Schulte, F.-U. Stein, T. Matsuyama, G. Jotzu, G. Meier, and A. Cavalleri, Light-induced anomalous Hall effect in graphene, Nat. Phys. 16, 38 (2020).

[55] B. Liu, H. Bromberger, A. Cartella, T. Gebert, M. Först, and A. Cavalleri, Generation of narrowband, high-intensity, carrier-envelope phase-stable pulses tunable between 4 and 18 THz, Opt. Lett. 42, 129 (2017).

[56] Y. Suemune, Thermal Conductivity of $\mathrm{BaTiO}_{3}$ and $\mathrm{SrTiO}_{3}$ from $4.5^{\circ}$ to $300^{\circ}$ K, J. Phys. Soc. Jpn. 20, 174 (1965).

[57] V. Fritsch, J. Hemberger, M. V. Eremin, H.-A. Krug von Nidda, F. Lichtenberg, R. Wehn, and A. Loidl, Magnetization and specific heat of $\mathrm{LaTiO}_{3}$, Phys. Rev. B 65, 212405 (2002).

[58] K. Kamarás, K. Barth, F. Keilmann, R. Henn, M. Reedyk, C. Thomsen, M. Cardona, J. Kircher, P. L. Richards, and
J. Stehlé, The low-temperature infrared optical functions of $\mathrm{SrTiO}_{3}$ determined by reflectance spectroscopy and spectroscopic ellipsometry, J. Appl. Phys. 78, 1235 (1995).

[59] F. Stern, Polarizability of a Two-Dimensional Electron Gas, Phys. Rev. Lett. 18, 546 (1967).

[60] A. McCollam, S. Wenderich, M. K. Kruize, V. K. Guduru, H. J. A. Molegraaf, M. Huijben, G. Koster, D. H. A. Blank, G. Rijnders, A. Brinkman, H. Hilgenkamp, U. Zeitler, and J. C. Maan, Quantum oscillations and subband properties of the two-dimensional electron gas at the $\mathrm{LaAlO}_{3} /$ $\mathrm{SrTiO}_{3}$ interface, APL Mater. 2, 022102 (2014).

[61] S. Thiel, G. Hammerl, A. Schmehl, C. W. Schneider, and J. Mannhart, Tunable quasi-two-dimensional electron gases in oxide heterostructures, Science 313, 1942 (2006).

[62] F. A. Rasmussen and K. S. Thygesen, Computational 2D materials database: electronic structure of transition-metal dichalcogenides and oxides, J. Phys. Chem. C 119, 13169 (2015).

[63] P. A. Gusikhin, V. M. Murav'ev, and I. V. Kukushkin, Observation of plasma waves with anomalously weak damping in a two-dimensional electron system, JETP Lett. 100, 648 (2015).

[64] T. Sakudo and H. Unoki, Dielectric properties of $\mathrm{SrTiO}_{3}$ at low temperatures, Phys. Rev. Lett. 26, 851 (1971).

[65] J. P. Carbotte, Properties of boson-exchange superconductors, Rev. Mod. Phys. 62, 1027 (1990).

[66] E. H. Hwang and S. Das Sarma, Dielectric function, screening, and plasmons in two-dimensional graphene, Phys. Rev. B 75, 205418 (2007).

[67] T. Ando, A. B. Fowler, and F. Stern, Electronic properties of two-dimensional systems, Rev. Mod. Phys. 54, 437 (1982).

[68] Z. Yan, Z. Peng, Z. Sun, J. Yao, Y. Zhu, Z. Liu, P. M. Ajayan, and J.M. Tour, Growth of bilayer graphene on insulating substrates, ACS Nano 5, 8187 (2011).

[69] J. Robertson, High dielectric constant oxides, Eur. Phys. J. Appl. Phys. 28, 265 (2004).

[70] E. McCann, D. S. L. Abergel, and V.I. Fal'ko, The low energy electronic band structure of bilayer graphene, Eur. Phys. J. Special Topics 148, 91 (2007).

[71] N. Reyren, S. Thiel, A. D. Caviglia, L. F. Kourkoutis, G. Hammerl, C. Richter, C. W. Schneider, T. Kopp, A.-S. Rüetschi, D. Jaccard, M. Gabay, D. A. Muller, J.-M. Triscone, and J. Mannhart, Superconducting interfaces between insulating oxides, Science 317, 1196 (2007).

[72] H. A. Hafez, X. Chai, A. Ibrahim, S. Mondal, D. Férachou, $\mathrm{X}$. Ropagnol, and T. Ozaki, Intense terahertz radiation and their applications, J. Opt. 18, 093004 (2016).

[73] K.-L. Yeh, M. C. Hoffmann, J. Hebling, and K. A. Nelson, Generation of $10 \mu \mathrm{J}$ ultrashort terahertz pulses by optical rectification, Appl. Phys. Lett. 90, 171121 (2007).

[74] M. C. Hoffmann, J. Hebling, H. Y. Hwang, K.-L. Yeh, and K. A. Nelson, THz-pump/THz-probe spectroscopy of semiconductors at high field strengths, J. Opt. Soc. Am. B 26, A29 (2009).

[75] C. Vicario, C. Ruchert, F. Ardana-Lamas, P. M. Derlet, B. Tudu, J. Luning, and C. P. Hauri, Off-resonant magnetization dynamics phase-locked to an intense phase-stable terahertz transient, Nat. Photonics 7, 720 (2013). 
[76] T. Kubacka et al., Large-amplitude spin dynamics driven by a $\mathrm{THz}$ pulse in resonance with an electromagnon, Science 343, 1333 (2014).

[77] F. Piazza and P. Strack, Quantum kinetics of ultracold fermions coupled to an optical resonator, Phys. Rev. A 90, 043823 (2014).

[78] R. Landig, L. Hruby, N. Dogra, M. Landini, R. Mottl, T. Donner, and T. Esslinger, Quantum phases from competing short- and long-range interactions in an optical lattice, Nature (London) 532, 476 (2016).

[79] A. Camacho-Guardian, R. Paredes, and S. F. CaballeroBenítez, Quantum simulation of competing orders with fermions in quantum optical lattices, Phys. Rev. A 96, 051602(R) (2017).

[80] J. Fan, X. Zhou, W. Zheng, W. Yi, G. Chen, and S. Jia, Magnetic order in a Fermi gas induced by cavity-field fluctuations, Phys. Rev. A 98, 043613 (2018).

[81] F. Schlawin and D. Jaksch, Cavity-Mediated Unconventional Pairing in Ultracold Fermionic Atoms, Phys. Rev. Lett. 123, 133601 (2019).

[82] D. Jaksch, S. A. Gardiner, K. Schulze, J. I. Cirac, and P. Zoller, Uniting Bose-Einstein Condensates in Optical Resonators, Phys. Rev. Lett. 86, 4733 (2001).

[83] P. Münstermann, T. Fischer, P. Maunz, P. W. H. Pinkse, and G. Rempe, Observation of Cavity-Mediated Long-Range Light Forces between Strongly Coupled Atoms, Phys. Rev. Lett. 84, 4068 (2000).

[84] C. Maschler and H. Ritsch, Cold Atom Dynamics in a Quantum Optical Lattice Potential, Phys. Rev. Lett. 95, 260401 (2005).
[85] K. Baumann, C. Guerlin, F. Brennecke, and T. Esslinger, Dicke quantum phase transition with a superfluid gas in an optical cavity, Nature (London) 464, 1301 (2010).

[86] R. Mottl, F. Brennecke, K. Baumann, R. Landig, T. Donner, and T. Esslinger, Roton-type mode softening in a quantum gas with cavity-mediated long-range interactions, Science 336, 1570 (2012).

[87] S. Zeytinoğlu, A. m. c. İmamoğlu, and S. Huber, Engineering Matter Interactions Using Squeezed Vacuum, Phys. Rev. X 7, 021041 (2017).

[88] F. Mivehvar, H. Ritsch, and F. Piazza, Superradiant Topological Peierls Insulator inside an Optical Cavity, Phys. Rev. Lett. 118, 073602 (2017).

[89] R. M. Kroeze, Y. Guo, V. D. Vaidya, J. Keeling, and B. L. Lev, Spinor Self-Ordering of a Quantum Gas in a Cavity, Phys. Rev. Lett. 121, 163601 (2018).

[90] V. D. Vaidya, Y. Guo, R. M. Kroeze, K. E. Ballantine, A. J. Kollár, J. Keeling, and B. L. Lev, Tunable-Range, PhotonMediated Atomic Interactions in Multimode Cavity QED, Phys. Rev. X 8, 011002 (2018).

[91] M. A. Norcia, R. J. Lewis-Swan, J. R. K. Cline, B. Zhu, A. M. Rey, and J. K. Thompson, Cavity-mediated collective spin-exchange interactions in a strontium superradiant laser, Science 361, 259 (2018).

[92] Y. Guo, R. M. Kroeze, V. D. Vaidya, J. Keeling, and B. L. Lev, Sign-Changing Photon-Mediated Atom Interactions in Multimode Cavity Quantum Electrodynamics, Phys. Rev. Lett. 122, 193601 (2019).

[93] F. Mivehvar, H. Ritsch, and F. Piazza, Cavity-QuantumElectrodynamical Toolbox for Quantum Magnetism, Phys. Rev. Lett. 122, 113603 (2019). 